\title{
Degree-Based Indices of Some Complex Networks
}

\author{
Lei Ding, ${ }^{1}$ Syed Ahtsham Ul Haq Bokhary $\mathbb{D}^{2},{ }^{2}$ Masood Ur Rehman $\mathbb{D}^{3}{ }^{3}$ Usman Ali $\mathbb{D}^{2}$ \\ Hirra Mubeen, ${ }^{2}$ Quaid Iqbal, ${ }^{4}$ and Jia-Bao Liu ${ }^{5}$ \\ ${ }^{1}$ School of Computer Engineering, Anhui Wenda University of Information Engineering, Hefei 231201, China \\ ${ }^{2}$ Centre for Advanced Studies in Pure and Applied Mathematics, Bahauddin Zakariya University, Multan, Pakistan \\ ${ }^{3}$ Department of Basic Sciences, Balochistan University of Engineering and Technology Khuzdar, Khuzdar 89100, Pakistan \\ ${ }^{4}$ Department of Mathematical Sciences, Fatima Jinnah Women University, Rawalpindi, Pakistan \\ ${ }^{5}$ School of Mathematics and Physics, Anhui Jianzhu University, Hefei 230601, Anhui, China \\ Correspondence should be addressed to Masood Ur Rehman; masood@mail.ustc.edu.cn
}

Received 26 January 2021; Revised 28 February 2021; Accepted 3 March 2021; Published 17 March 2021

Academic Editor: Ahmet Sinan Cevik

Copyright $\odot 2021$ Lei Ding et al. This is an open access article distributed under the Creative Commons Attribution License, which permits unrestricted use, distribution, and reproduction in any medium, provided the original work is properly cited.

A topological index is a numeric quantity assigned to a graph that characterizes the structure of a graph. Topological indices and physico-chemical properties such as atom-bond connectivity (ABC), Randić, and geometric-arithmetic index (GA) are of great importance in the QSAR/QSPR analysis and are used to estimate the networks. In this area of research, graph theory has been found of considerable use. In this paper, the distinct degrees and degree sums of enhanced Mesh network, triangular Mesh network, star of silicate network, and rhenium trioxide lattice are listed. The edge partitions of these families of networks are tabled which depend on the sum of degrees of end vertices and the sum of the degree-based edges. Utilizing these edge partitions, the closed formulae for some degree-based topological indices of the networks are deduced.

\section{Introduction and Preliminary Results}

A molecular graph is a representation of the structural formula of a chemical compound in terms of graph theory, whose vertices correspond to the atoms of the compound and edges correspond to chemical bonds between atoms.

In the modern age, network structures have great significance in the field of chemistry, information technology, communication, and physical structures. Each network can be distinguished by a numeric quantity associated with it by defined rules under certain parameters. These rules are known as topological indices. Any numerical value allocated to a graph, which classifies the structure of a graph, is called a topological index. Popular and well-studied types of topological indices are degree-based topological indices, distance-based topological indices, and counting-related polynomials and indices of graphs. Degree-based topological indices are of great importance among these groups and play a strong role in chemical graph theory and in chemistry. More specifically, to guess the biological activity of various chemical compounds, these topological indices are used. In the evaluation of quantitative structure-activity (QSAR) and structure-property (QSPR), topological indices and physicochemical properties such as atom-bond connectivity (ABC), Wiener index, Szeged index, Randić index, Zagreb indices [1], and geometric-arithmetic (GA) index are of great importance which are used to guess the bioactivity and properties of various chemical compounds.

The Wiener index is originally the first and most studied topological index. It was the first molecular topological index that was used in chemistry. Wiener shows that the Wiener index number is closely correlated with the boiling points of alkane molecules [2]. Later, work on the quantitative structure-activity relationships showed that it is also correlated with other quantities including the parameters of its critical point, the density, surface tension, and viscosity of its liquid phase, and the Van der Waals surface area of the molecule. After the Wiener index, the theory of topological indices began. In mathematical chemistry, there is a huge amount of topological indices of the form 


$$
\operatorname{TI}(G)=\sum_{v_{i} v_{j} \in V(G)} F\left(d_{i}, d_{j}\right),
$$

where $d_{i}$ is the degree of each $v_{i}$ and $F$ is a pertinently picked function with the characteristic $F(x, y)=F(y, x)$. A huge amount of topological indices were introduced by various chemists in the advanced studies of indices. For different molecular families, several researchers have shown different computational and theoretical results related to certain topological indices and related them with energies of the graphs. If $f_{1}, f_{2}, \ldots, f_{n}$ are the eigenvalues of the matrix TI, then energy can be defined as

$$
\varepsilon_{\mathrm{TI}}(G)=\sum_{1 \leq i \leq n}\left|f_{i}\right| .
$$

The most extensively studied graph energy is the Randic index. J. Rad et al. [3] analyzed the energy (Zagreb energy) and Estrada (Zagreb Estrada) index of a graph, and both are based on the Zagreb matrix's eigenvalues. Furthermore, for these new graph invariants, they define upper and lower limits and relationships between them. The relationship between the Kirchhoff index and Laplaçian graph energy is introduced by Das et al. [4]. Milovanovic et al. [5] gave some lower bound for Kirchhoff index as well some new lower bounds for the Laplacian energy of a graph in the same article. For any connected graph $G$, Bozkurt et al. [6] acquired an upper bound for distance energy. For the distance energy of connected diameter 2 graphs with given numbers of vertices and edges, they gave an upper bound. In addition, they also provide a lower bound for the distance energy of unicyclic graphs having odd girth. Alikhani et al. [7] compute the $\mathrm{ABC}$ index for some families of nanostars and polyphenylene dendrimers. Bača et al. [8] studied the geometric-arithmetic (GA) indices of carbon nanotube networks and fullerene networks. Baig et al. $[9,10]$ computed Omega, Sadhana, and Pl polynomials, for Benzoid nanotubes for the first time. For these interconnection networks, Baig et al. [11] compute the first general indices of Zagreb, $\mathrm{ABC}, \mathrm{GA}, \mathrm{ABC}_{4}$, and $\mathrm{GA}_{5}$ and give closed formulae of these indices.

In pairs of past decays, the use of graph theoretical methods to explain the chemical structure has gained more and more importance. Since the early periods in which such formalism was used to predict simple properties on simple molecules, such as alkane boiling points, up to the design of novel lead anticancer drugs, for example, considerable progress was made and a long path was covered.

In this paper, by graph $G$, we always mean the network having vertex set $V(G)$ and edge set $E(G)$. We represent the degree of $u \in V(G)$ by $d_{u}$ and $S_{u}=\sum_{v \in N_{G}(u)} d_{v}$, where $N_{G}(u)=\{v \in V(G) \mid u v \in E(G)\}$. We are using notations in the present paper from $[12,13]$. The atom-bond connectivity $(\mathrm{ABC})$ is the well-known degree-based topological index, which is introduced by Estrada et al. in [14] and defined as

$$
A B C(G)=\sum_{u v \in E(G)} \sqrt{\frac{d_{u}+d_{v}-2}{d_{u} d_{v}}} .
$$

Vukičević et al., in [15], introduced a well-known connectivity topological descriptor is geometric-arithmetic (GA) index and defined

$$
\mathrm{GA}(G)=\sum_{u v \in E(G)} \frac{2 \sqrt{d_{u} d_{v}}}{d_{u}+d_{v}} .
$$

If we can find the edge partition of these interconnection chemical networks based on the sum of the degrees of the end vertices of each edge in these graphs, only $\mathrm{ABC}_{4}$ and $\mathrm{GA}_{5}$ indices can be computed. The fourth version of $\mathrm{ABC}$ index is introduced by Ghorbani et al. [16] and is given as follows:

$$
\mathrm{ABC}_{4}(G)=\sum_{u v \in E(G)} \sqrt{\frac{S_{u}+S_{v}-2}{S_{u} S_{v}}} .
$$

The fifth version of GA index is introduced by Graovac et al. [17] and is given as follows:

$$
\mathrm{GA}_{5}(G)=\sum_{u v \in E(G)} \frac{2 \sqrt{S_{u} S_{v}}}{S_{u}+S_{v}} .
$$

Because of their great importance in chemistry, these topological indices are extensively studied by various mathematicians. Hayat and Imran studied $\mathrm{ABC}_{4}$ and $\mathrm{GA}_{5}$ and gave close formulae of these indices for some nanotubes and their corresponding nanotori. They also give a characterization of $k$-regular graphs with respect to their $\mathrm{GA}_{5}$ [18]. In [19], Hayat and Imran compute the (ABC), (GA), and Zagreb indices of $V C_{5} C_{7}[p, q], H C_{5} C_{7}[p, q]$ and $S C_{5} C_{7}[p, q]$ nanotubes. They also compute $\mathrm{ABC}_{4}$ and $\mathrm{GA}_{5}$ for these nanotubes. Similarly, they compute the $\left(\mathrm{ABC}_{4}\right)$ and $\left(\mathrm{GA}_{5}\right)$ indices of $\mathrm{H}$-naphtalenic nanotubes and chain silicate, silicate, oxide, hexagonal, and honeycomb networks in [20,21].

This paper deals with a specific organization form of matter. Other forms and description are given and discussed by different authors. For example, for the first time, Ali and Mehdi compute the GA index of TUZC6[p,q], TUAC6 $[p, q], H C 5 C 7[p, q]$, and SC5C7 $[p, q]$ nanotubes in [22]. In [23], W. Lin et al. disprove Dimitrov's "mo du lo7conjecture." Shang established new lower bounds for the Gaussian Estrada index in terms of the first Zagrab index and the number of vertices and edges in [24]. Also, Shang obtained the upper and lower bounds for the Laplacian Estrada index of $\Gamma(G)$ based on the vertex degrees of the graph $G$ in [25]. The rest of the paper breaks as follows. Sections 2-5 contain the degree-based topological indices of enhanced mesh, triangular mesh, star of silicate network, and rhenium trioxide lattice, respectively. In Section 6, we give the conclusion of the paper and pose some open problems. Throughout this paper, $\left(d_{u}, d_{v}\right)$ represents the number of edges of the given graph $G$ with end vertices of each edge having degrees $d_{u}$ and $d_{v}$, respectively. Similarly, $\left(S_{u}, S_{v}\right)$ denotes the number of edges of the given graph $G$ with end vertices of each edge having degree sum $S_{u}$ and $S_{v}$, respectively. By degree sum $S_{u}$, it is meant to be the sum of degrees of all vertices adjacent to the vertex $u$. 


\section{Enhanced Mesh}

In this section, we study the degree-based topological descriptors of enhanced mesh network [26].

2.1. Construction. The graph whose vertices correspond to the points in the plane with integer coordinates, $x$-coordinates in the range $1,2, \ldots, n$ and $y$-coordinates in the range $1,2, \ldots, m$ and two vertices are connected by an edge whenever the corresponding points are at distance 1 , is a common form of lattice graph. In other words, for the point set mentioned, it is a unit distance graph. The term $n$-mesh has also been given to various other types of graphs with a certain structure in the literature, such as the Cartesian product of a number of path graphs. The Cartesian product of paths of order $a_{1}, a_{2}, \ldots, a_{n}$ is an $n$-mesh $M\left(a_{1}, a_{2}, \ldots, a_{n}\right)$, which is defined as

$$
M\left(a_{1}, a_{2}, \ldots, a_{n}\right)=P_{a_{1}} \times P_{a_{2}} \times \cdots \times P_{a_{n}} .
$$

For $n$-mesh, $M\left(a_{1}, a_{2}, \ldots, a_{n}\right)$ has order

$$
\left|V\left(M\left(a_{1}, a_{2}, \ldots, a_{n}\right)\right)\right|=a_{1}, a_{2}, \ldots, a_{n}
$$

and size

$$
E\left(M\left(a_{1}, a_{2}, \ldots, a_{n}\right)\right)=a_{1}, a_{2}, \ldots, a_{n}\left(n-\frac{1}{a_{1}}-\frac{1}{a_{2}}-\cdots-\frac{1}{a_{n}}\right) .
$$

Here, we are going to discuss the enhance 2-mesh network. A 2-mesh $M\left(P_{a_{1}} \times P_{a_{2}}\right)$ has vertex set

$$
V=\{(i, j): 1 \leq i \leq m, 1 \leq j \leq n\}
$$

and the set

$$
E=\{((i, j),(i, j+1)): 1 \leq i \leq m, 1 \leq j \leq n-1\} \cup\{((i, j),(i+1, j)): 1 \leq i \leq m-1,1 \leq j \leq n\}
$$

is an edge set. An enhanced mesh $\operatorname{EM}\left(P_{a_{1}} \times P_{a_{2}}\right)$ is resulted by replacing each 4-cycle of $M\left(P_{a_{1}} \times P_{a_{2}}\right)$ by a wheel $W_{4}$ on 4 vertices. Thus, a wheel $W_{4}$ is a graph retrieved by joining the central vertex to each vertex of cycle $C_{4}$. The hub (central vertex) of $W_{4}$ is a new vertex.

Assume that $h_{i j}, 1 \leq i \leq m-1,1 \leq j \leq n-1$, is the collection of all hub (central) vertices.

Theorem 1. For $m, n \geq 5$ and $\alpha, \beta=\gamma, c \in R$, the $A B C$ index of enhanced mesh $\operatorname{EM}\left(P_{a_{1}} \times P_{a_{2}}\right)$ is $\operatorname{ABC}\left(\operatorname{EM}\left(P_{a_{1}} \times P_{a_{2}}\right)\right)=$ $\alpha m n+\beta m+\gamma n+c \approx 3.1715 m n-2.2641(m+n)+1.7492$.

Proof. Suppose that $G$ is a graph of enhanced mesh. The set of all distinct degrees $d_{u}$ for $u \in V(\operatorname{EM}(m, n))$ is $\{3,4,5,8\}$. From Figure 1, we see that the number of edges of type $(3,4)$ and $(3,5)$ are 4 and 8 , respectively.

Every vertex that is lying on the boundary of the graph $\operatorname{EM}(m, n)$, except the corner vertices, are of degree 5 and the oblique edges which are adjacent to the vertex of degree 4 are edges of type $(4,5)$. There are total $2(m-2)+2(n-2)=$ $2 m+2 n-8=2(m+n-4)$ vertices on the boundary of degree 5 , and each vertex induces two edges of type $(4,5)$. Thus, the number of edges of type $(4,5)$ is $4(m+n-4)$.
The central vertex of each wheel graph $W_{4}$ is of degree 4 . This implies that there are total $(m-1)(n-1)$ vertices of degree 4 . The 4 corner vertices of degree 4 induces one edge of type $(4,8)$, and the remaining $2(m-3)+2(n-3)=2$ $(m+n-6)$ vertices lying adjacent to the boundary vertices induces 2 edges of type $(4,8)$. Each of the remaining $(m-$ $3)(n-3)$ vertices of degree 4 induces 4 edges of type $(4,8)$. Thus, the number of edges of type $(4,8)$ is $4+4(m+n-$ $6)+4(m-3)(n-3)=4(m n-2 m-2 n+4)$.

There are total $2(m+n-4)$ vertices on the boundary of the graph $G$ which are of degree 5 . Each vertex induces one edge of type $(5,8)$ and two edges of type $(4,5)$. Thus, the number of edges of type $(5,8)$ are $4(m+n-4)$. Furthermore, there are total $4(m+n-6)$ edges of type $(5,5)$. This edge partition of enhanced mesh based on the degrees of end vertices is shown in Table 1.

Now, by using this edge partition, we compute the ABC index of enhanced mesh as follows:

$$
\operatorname{ABC}(G)=\sum_{u v \in E(G)} \sqrt{\frac{d_{u}+d_{v}-2}{d_{u} d_{v}}} .
$$

This implies that

$$
\begin{aligned}
A B C(G)= & 4 \sqrt{\frac{3+4-2}{3 \cdot 4}}+8 \sqrt{\frac{3+5-2}{3 \cdot 5}}+4(m+n-4) \sqrt{\frac{4+5-2}{4 \cdot 5}}+4(m n-2 m-2 n+4) \sqrt{\frac{4+8-2}{4 \cdot 8}} \\
& +2(m+n-6) \sqrt{\frac{5+5-2}{5 \cdot 5}}+2(m+n-4) \sqrt{\frac{5+8-2}{5 \cdot 8}}+(2 m n-5 m-5 n+12) \sqrt{\frac{8+8-2}{8 \cdot 8}}
\end{aligned}
$$




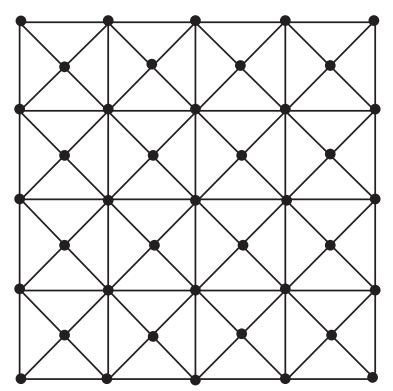

FIgURE 1: Enhanced mesh $M(5,5)$ network.

TABLE 1: $\operatorname{EM}(m, n)$ edge partition centred on the degree of the ending vertices of each edge, where $u v \in E(G)$.

\begin{tabular}{lc}
\hline$\left(d_{u}, d_{v}\right)$ & Number of edges \\
\hline$(3,4)$ & 4 \\
$(3,5)$ & 8 \\
$(4,5)$ & $4(m+n-4)$ \\
$(4,8)$ & $4(m n-2 m-2 n+4)$ \\
$(5,5)$ & $2(m+n-6)$ \\
$(5,8)$ & $2(m+n-4)$ \\
$(8,8)$ & $(2 m n-5 m-5 n+12)$ \\
\hline
\end{tabular}

After simplification, we obtain

$$
\begin{aligned}
A B C(E M(m, n))= & \left(\sqrt{5}+\frac{\sqrt{7}}{2 \sqrt{2}}\right) m n+\left(\frac{4 \sqrt{2}}{5}+2 \sqrt{\frac{7}{5}}+\sqrt{\frac{11}{10}}-2 \sqrt{5}-\frac{5 \sqrt{7}}{4 \sqrt{2}}\right) m+\left(\frac{4 \sqrt{2}}{5}+2 \sqrt{\frac{7}{5}}+\sqrt{\frac{11}{10}}-2 \sqrt{5}-\frac{5 \sqrt{7}}{4 \sqrt{2}}\right) n \\
& +\left(2 \sqrt{\frac{5}{3}}+8 \sqrt{\frac{2}{5}}-\frac{24 \sqrt{2}}{5}-8 \sqrt{\frac{7}{5}}-4 \sqrt{\frac{11}{10}}+4 \sqrt{5}+3 \sqrt{\frac{7}{2}}\right) \\
\approx & 3.1715 m n-2.2641(m+n)+1.7492
\end{aligned}
$$

We will compute the GA index of enhanced mesh, in the following result.

Theorem 2. For any $m, n \geq 5$ and $\eta, v=\mu, c_{1} \in R$, the $G A$ index of enhanced mesh $\operatorname{EM}\left(P_{a_{1}} \times P_{a_{2}}\right)$ is $\operatorname{GA}\left(\operatorname{EM}\left(P_{a_{1}} \times P_{a_{2}}\right)\right)$ $=\eta m n+\nu m+\mu n+c_{1} \approx 6.7712 m n-4.6212(m+n)+11.8948$.

Proof. Using edge partition given in Table 1 and the formula $\sum_{u v \in E(G)} 2\left(\sqrt{d_{u} d_{v}} / d_{u}+d_{v}\right)$, we get the proof of the statement.

The $\mathrm{ABC}_{4}$ and $\mathrm{GA}_{5}$ indices of the enhanced mesh is computed in the next two results.

Theorem 3. For $m, n \geq 5$ and $\zeta, \varsigma=\tau, c_{2} \in R$, the $A B C_{4}(G)$ index of enhanced mesh $\operatorname{EM}\left(P_{a_{1}} \times P_{a_{2}}\right)$ is $A B C_{4}\left(E M\left(P_{a_{1}} \times\right.\right.$
$\left.\left.P_{a_{2}}\right)\right)=\zeta m n+\varsigma m+\tau n+c_{2} \approx 1.3054 m n-0.9559(m+n)-$ 1.6792 .

Proof. Suppose that $G$ is a graph of enhanced mesh. The corner vertices have degree sum 14 . The corner hub vertex have degree sum 21 . The vertices adjacent to corner vertices have degree sum 24 . The remaining vertices lying on the boundaries of the graph and the adjacent hub vertices (except the corner hub vertices) has degree sum 26 . The reminiscing hub vertices has degree sum 32 . The 8 degree vertices adjacent to the corner hub has degree sum 42 . The 8 degree vertices adjacent to the corner vertices lying on the boundary of graph have degree sum 45. All the remaining 8 degree vertices has degree sum 48 . Thus, the set of all distinct degree sums $S_{u}$ for $u \in V(\operatorname{EM}(m, n))$ is $\{14,21,24,26,32,42,45,48\}$. Using this information, the edge 
TABLE 2: Edge partition of $\operatorname{EM}(m, n)$ on the basis of the sum of vertices which is a distance unit from the end vertices of each edge.

\begin{tabular}{lc}
\hline$\left(S_{u}, S_{v}\right)$ where $u v \in E(G)$ & Number of edges \\
\hline$(14,21)$ & 4 \\
$(14,24)$ & 8 \\
$(21,24)$ & 8 \\
$(21,42)$ & 4 \\
$(24,26)$ & 16 \\
$(24,42)$ & 8 \\
$(26,26)$ & $2(3 m+3 n-26)$ \\
$(26,42)$ & 8 \\
$(26,45)$ & $6(m+n-8)$ \\
$(32,42)$ & 4 \\
$(32,45)$ & $4(m+n-8)$ \\
$(32,48)$ & $4(m n-4 m-4 n+16)$ \\
$(42,45)$ & 8 \\
$(45,45)$ & $2(m+n-10)$ \\
$(45,48)$ & $2(m+n-8)$ \\
$(48,48)$ & $(2 m n-9 m-9 n+40)$ \\
\hline
\end{tabular}

Now, we compute the formula for $\mathrm{ABC}_{4}$ index for $G$ by using the edge partition given in Table 2 , since

$$
\mathrm{ABC}_{4}(G)=\sum_{u v \in E(G)} \sqrt{\frac{S_{u}+S_{v}-2}{S_{u} S_{v}}} .
$$

This implies that

partition of the graph $G$ shown in Figure 1 is computed in Table 2.

$$
\begin{aligned}
\mathrm{ABC}_{4}(G)= & 4 \sqrt{\frac{14+21-2}{14 \cdot 21}}+8 \sqrt{\frac{14+24-2}{14 \cdot 24}}+8 \sqrt{\frac{21+24-2}{21 \cdot 24}}+4 \sqrt{\frac{21+42-2}{21 \cdot 42}} \\
& +16 \sqrt{\frac{24+26-2}{24 \cdot 26}}+8 \sqrt{\frac{24+42-2}{24 \cdot 42}}+2(3 m+3 n-26) \sqrt{\frac{26+26-2}{26 \cdot 26}}+8 \sqrt{\frac{26+42-2}{26 \cdot 42}} \\
& +6(m+n-8) \sqrt{\frac{26+45-2}{26 \cdot 45}}+4 \sqrt{\frac{32+42-2}{32 \cdot 42}}+4(m+n-8) \sqrt{\frac{32+45-2}{32 \cdot 45}+4(m n-4 m-4 n+16)} \\
& \sqrt{\frac{32+48-2}{32 \cdot 48}}+8 \sqrt{\frac{42+45-2}{42 \cdot 45}}+2(m+n-10) \sqrt{\frac{45+45-2}{45 \cdot 45}}+2(m+n-8) \sqrt{\frac{45+48-2}{45 \cdot 48}} \\
& +(2 m n-9 m-9 n+40) \sqrt{\frac{48+48-2}{48 \cdot 48}}
\end{aligned}
$$

After a simple calculation, the above equation can be reduced as

$$
\begin{aligned}
& \mathrm{ABC}_{4}(G)=\left(\frac{\sqrt{13}}{4}+\frac{\sqrt{47}}{12 \sqrt{2}}\right) m n+\left(\frac{30}{13 \sqrt{2}}+6 \sqrt{\frac{13}{390}}-\sqrt{13}+\frac{\sqrt{91}}{6 \sqrt{15}}+\sqrt{\frac{5}{6}}-\frac{3 \sqrt{47}}{8 \sqrt{2}}+\frac{4 \sqrt{22}}{45}\right) m \\
& +\left(\frac{30}{13 \sqrt{2}}+6 \sqrt{\frac{13}{390}}-\sqrt{13}+\frac{\sqrt{91}}{6 \sqrt{15}}+\sqrt{\frac{5}{6}}-\frac{3 \sqrt{47}}{8 \sqrt{2}}+\frac{4 \sqrt{22}}{45}\right) n+ \\
& \left(4 \sqrt{\frac{3}{7}}+\frac{4 \sqrt{11}}{7 \sqrt{2}}+16 \sqrt{\frac{1}{13}}+\frac{4 \sqrt{43}}{3 \sqrt{14}}-20 \sqrt{\frac{1}{2}}+8 \sqrt{\frac{11}{182}}+\frac{4 \sqrt{61}}{21 \sqrt{2}}+\frac{8 \sqrt{17}}{3 \sqrt{42}}\right. \\
& \left.-48 \sqrt{\frac{23}{390}}+2 \sqrt{\frac{3}{14}}+4 \sqrt{13}-\frac{4 \sqrt{91}}{3 \sqrt{15}}-8 \sqrt{\frac{5}{6}}+\frac{5 \sqrt{47}}{3 \sqrt{2}}-\frac{40 \sqrt{22}}{45}\right) \\
& \approx 1.3054 m n-0.9559(m+n)-1.6792 \text {. }
\end{aligned}
$$


The following theorem gives the $\mathrm{GA}_{5}$ index of enhanced mesh $M(m, n)$.

Theorem 4. For $m, n \geq 5$ and $\kappa, \lambda=\sigma, c_{3} \in R$, the $G A_{5}(G)$ index of enhanced mesh $\operatorname{EM}\left(P_{a_{1}} \times P_{a_{2}}\right)$ is $G A_{5}\left(E M\left(P_{a_{1}} \times\right.\right.$ $\left.\left.P_{a_{2}}\right)\right)=\kappa m n+\lambda m+\sigma n+c_{3} \approx 4.9192 m n+25.6994(m+n)+$ 3.7338 .

Proof. Following the information given in Table 2 and the formula $\sum_{u v \in E(G)} 2\left(\sqrt{S_{u} S_{v}} / S_{u}+S_{v}\right)$, we easily get the required proof.

\section{Triangular Mesh}

In this section, we are going to study the degree-based topological descriptors for the triangular mesh network [26].
We denote the radix $-n$ triangular mesh network by $T_{n}$ having node set

$$
V\left(T_{n}\right)=\{(x, y) \mid 0 \leq x, y \leq n \text { and } 0 \leq x+y \leq n\},
$$

and there exists a mesh arc between nodes $\left(x_{1}, y_{1}\right)$ and $\left(x_{2}, y_{2}\right)$ if $\left|x_{1}-x_{2}\right|+\left|y_{1}-y_{2}\right| \leq n-1$ and $x_{1}+y_{1} \leq x_{2}+y_{2}$. The number of vertices (nodes) in a $T_{n}$ is $n(n+1) / 2$. The degree of node in the aforementioned network may be 2, 4, or 6 . There exist three vertices of degree 2 , which we call as corner vertices. Throughout this section, we represent $G$ by the graph of triangular mesh network $T_{n}$. The graph of triangular mesh $T_{5}$ is shown in Figure 2.

Theorem 5. For $n \geq 4$, the $A B C$ index of $G$ is

$$
\operatorname{ABC}\left(T_{n}\right)=\left(\frac{\sqrt{5}}{2 \sqrt{2}}\right) n^{2}+\left(\frac{3 \sqrt{3}}{2 \sqrt{2}}+6 \sqrt{\frac{1}{3}}-\frac{7 \sqrt{5}}{2 \sqrt{2}}\right) n+\left(6 \sqrt{\frac{1}{2}}-3 \sqrt{\frac{3}{2}}-18 \sqrt{\frac{1}{3}}+6 \sqrt{\frac{5}{2}}\right) \approx 0.7906 n^{2}-0.2328 n-0.3371
$$

Proof. The set of all distinct degrees $d_{u}$ for $u \in V(G)$ is $\{2,4,6\}$. The edge partition of the graph $G$ based on the

$$
\operatorname{ABC}(G)=\sum_{u v \in E(G)} \sqrt{\frac{d_{u}+d_{v}-2}{d_{u} d_{v}}}
$$
degrees of the end vertices lying at distance one from the end vertices of each edge is shown in Table 3.

By using the edge partition above, we calculate the triangular mesh ABC index as follows:

$$
\mathrm{ABC}(G)=6 \sqrt{\frac{2+4-2}{2 \cdot 4}}+3(n-2) \sqrt{\frac{4+4-2}{4 \cdot 4}}+6(n-3) \sqrt{\frac{4+6-2}{4 \cdot 6}}+\frac{3\left(n^{2}-7 \cdot n+12\right)}{2} \cdot \sqrt{\frac{6+6-2}{6 \cdot 6}} .
$$

After simplification, we obtain

$$
\operatorname{ABC}\left(T_{n}\right)=\left(\frac{\sqrt{5}}{2 \sqrt{2}}\right) n^{2}+\left(\frac{3 \sqrt{3}}{2 \sqrt{2}}+6 \sqrt{\frac{1}{3}}-\frac{7 \sqrt{5}}{2 \sqrt{2}}\right) n+\left(6 \sqrt{\frac{1}{2}}-3 \sqrt{\frac{3}{2}}-18 \sqrt{\frac{1}{3}}+6 \sqrt{\frac{5}{2}}\right) \approx 0.7906 n^{2}-0.2328 n-0.3371
$$

We will compute the GA index of the triangular mesh in Theorem 6. For $n \geq 4$, the following theorem.

$$
\mathrm{GA}(G)=3 n^{2}+\left(\frac{12 \sqrt{6}}{5}-18\right) n+\left(4 \sqrt{2}-\frac{36 \sqrt{6}}{5}-42\right) \approx 3 n^{2}-12.1212 n-53.9795
$$

Proof. The result is followed by Table 3, and $\sum_{u v \in E(G)} 2\left(\sqrt{d_{u} d_{v}} /\left(d_{u}+d_{v}\right)\right)$.

The three corner vertices has degree sum 8 . The 6 vertices adjacent to the corner vertices has degree sum 16 . The remaining $3(n-4)$ vertices of degree 4 has degree sum 20 . The three corner vertices of degree 6 has degree sum 32. The remaining $((n-3)(n-2) / 2)-3$ vertices adjacent to the vertices of degree 4 has degree sum 32. All the remaining vertices of degree 6 has degree sum 36. Thus, the set of all distinct degree sums $S_{u}$ for $u \in V\left(T_{n}\right)$ is $\{8,16,20,28,32,36\}$. In the following table, the edge partition is given.

The indices $\mathrm{ABC}_{4}$ and $\mathrm{GA}_{5}$ of the triangular mesh is computed in the next two theorems. 


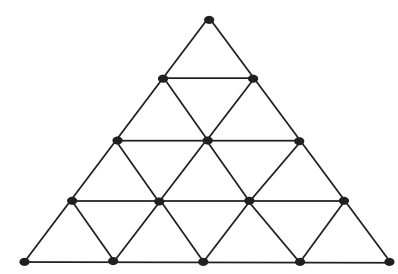

FIgURE 2: Triangular mesh $T_{5}$ network.

Table 3: The $T_{n}$ edge partition depends on the degree of the end vertices of each edge, where $u v \in E(G)$.

\begin{tabular}{lc}
\hline$\left(d_{u}, d_{v}\right)$ & Number of edges \\
\hline$(2,4)$ & 6 \\
$(4,4)$ & $3(n-2)$ \\
$(4,6)$ & $6(n-3)$ \\
$(6,6)$ & $\left(3\left(n^{2}-7 n+12\right) / 2\right)$ \\
\hline
\end{tabular}

TABLE 4: Edge partition of $T_{n}$ centred on the number of the vertices of the degree lying at distance 1 from the end vertices of each edge.

\begin{tabular}{lc}
\hline$\left(S_{u}, S_{v}\right)$ where $u v \in E(G)$ & Number of edges \\
\hline$(8,16)$ & 6 \\
$(16,16)$ & 3 \\
$(16,20)$ & 6 \\
$(16,28)$ & 6 \\
$(20,20)$ & $3(n-5)$ \\
$(20,28)$ & 6 \\
$(20,32)$ & $6(n-5)$ \\
$(28,32)$ & 6 \\
$(32,32)$ & $3(n-5)$ \\
$(32,36)$ & $6(n-6)$ \\
$(36,36) k$ & $\left(3\left(n^{2}-13 n+42\right) / 2\right)$ \\
\hline
\end{tabular}

Theorem 7. The $A B C_{4}$ index of the graph $G$, for $n \geq 8$, is computed as

$$
\begin{aligned}
\mathrm{ABC}_{4}(G)= & \frac{\sqrt{35}}{12 \sqrt{2}} n^{2}+\left(\frac{3 \sqrt{5}}{4}+\frac{3 \sqrt{31}}{16 \sqrt{2}}+\frac{3 \sqrt{19}}{10 \sqrt{2}}+\frac{3 \sqrt{11}}{4 \sqrt{3}}-\frac{13 \sqrt{35}}{12 \sqrt{2}}\right) n \\
& +\left(\frac{3 \sqrt{11}}{4}+\frac{3 \sqrt{15}}{8 \sqrt{2}}+\frac{3 \sqrt{17}}{2 \sqrt{10}}+\frac{3 \sqrt{3}}{2 \sqrt{2}}+3 \sqrt{\frac{23}{70}}-\frac{15 \sqrt{5}}{4}-\frac{15 \sqrt{31}}{16 \sqrt{2}}+\frac{3 \sqrt{29}}{4 \sqrt{7}}-\frac{3 \sqrt{19}}{2 \sqrt{2}}-\frac{9 \sqrt{11}}{2 \sqrt{3}}+\frac{7 \sqrt{35}}{2 \sqrt{2}}\right) \\
\approx & 0.3486 n^{2}+0.2441 n-3.5606 .
\end{aligned}
$$

Proof. We use the information given in Table 4 to compute This implies that the formula for $\mathrm{ABC}_{4}$ index for $G$.

$$
\operatorname{ABC}_{4}(G)=\sum_{u v \in E(G)} \sqrt{\frac{S_{u}+S_{v}-2}{S_{u} S_{v}}} .
$$

$$
\begin{aligned}
\mathrm{ABC}_{4}(G)= & 6 \sqrt{\frac{8+16-2}{8 \cdot 16}}+3 \sqrt{\frac{16+16-2}{16 \cdot 16}}+6 \sqrt{\frac{16+20-2}{16 \cdot 20}}+6 \sqrt{\frac{16+28-2}{16 \cdot 28}}+3(n-5) \sqrt{\frac{20+20-2}{20 \cdot 20}} \\
& +6 \sqrt{\frac{20+28-2}{20 \cdot 28}}+6(n-5) \sqrt{\frac{20+32-2}{20 \cdot 32}}+6 \sqrt{\frac{28+32-2}{28 \cdot 32}+3(n-5) \sqrt{\frac{32+32-2}{32 \cdot 32}}} \\
& +6(n-6) \sqrt{\frac{32+36-2}{32 \cdot 36}}+\left(\frac{3\left(n^{2}-13 n+42\right)}{2}\right) \sqrt{\frac{36+36-2}{36 \cdot 36}} .
\end{aligned}
$$

After a simple calculation, the above equation can be reduced as 


$$
\begin{aligned}
\mathrm{ABC}_{4}(G)= & \frac{\sqrt{35}}{12 \sqrt{2}} n^{2}+\left(\frac{3 \sqrt{5}}{4}+\frac{3 \sqrt{31}}{16 \sqrt{2}}+\frac{3 \sqrt{19}}{10 \sqrt{2}}+\frac{3 \sqrt{11}}{4 \sqrt{3}}-\frac{13 \sqrt{35}}{12 \sqrt{2}}\right) n \\
& +\left(\frac{3 \sqrt{11}}{4}+\frac{3 \sqrt{15}}{8 \sqrt{2}}+\frac{3 \sqrt{17}}{2 \sqrt{10}}+\frac{3 \sqrt{3}}{2 \sqrt{2}}+3 \sqrt{\frac{23}{70}}-\frac{15 \sqrt{5}}{4}-\frac{15 \sqrt{31}}{16 \sqrt{2}}+\frac{3 \sqrt{29}}{4 \sqrt{7}}-\frac{3 \sqrt{19}}{2 \sqrt{2}}-\frac{9 \sqrt{11}}{2 \sqrt{3}}+\frac{7 \sqrt{35}}{2 \sqrt{2}}\right) \\
\approx & 0.3486 n^{2}+0.2441 n-3.5606 .
\end{aligned}
$$

Theorem 8. For $n \geq 8$, we have

$$
\begin{aligned}
\mathrm{GA}_{5}(G)= & \left(\frac{3}{2}\right) n^{2}+\left(\frac{24 \sqrt{10}}{13}-\frac{27}{2}\right) n+ \\
& \left(4 \sqrt{2}+\frac{8 \sqrt{5}}{3}+\frac{24 \sqrt{7}}{11}+\sqrt{35}-\frac{120 \sqrt{10}}{13}+\frac{8 \sqrt{14}}{5}+\frac{72 \sqrt{2}}{17}+36\right) \approx 1.5 n^{2}-7.6619 n+42.0943
\end{aligned}
$$

Proof. Table 4 and formula $\sum_{u v \in E(G)} 2\left(\sqrt{S_{u} S_{v}} / S_{u}+S_{v}\right)$ supply the proof of the statement.

\section{Star of Silicate Network}

In the present section, we explore the degree-based topological descriptors for the star of silicate network [27].

We define the creation of a new star of a silicate network from the star of the David network in Figure 3.

Step1: construct a star of David graph $H$ of dimension 1 (Figure 3).

Step2: by inserting $2 n-2$ vertices at every edge of $H$, divide each edge into $2 n-1$ edges.

Step3: if one is the mirror image of the other and if they are at an odd distance $1,3,5,7, \ldots,(6 n-1)$ from one corner vertices except the pairs at a distance $(2 n-1)$, connect any two vertices $u$ and $v$ by an edge.

Step4: at each new crossing of the edge, insert a new vertex. The resulting network is called the n-dimensional star of David network, which is denoted by $\operatorname{SD}(n)$.

Step5: replacing each $K 3$ subgraph with a tetrahedron. This network is known as the $n$ dimension star silicate star or star of silicate network star and is denoted by $\operatorname{SSL}(n)$. The graph for the SSL (2) silicate network star is shown in Figure 4.

Throughout this section, we denote the graph of star silicate network SSL $(n)$ by $G$.

In the graph of star of silicate network, the 6 corner vertices and the central vertex of each tetrahedron is of degree 3. The vertices inserted in step two of the construction have degree 4 . All the remaining vertices have degree 6 . Thus, the set of all distinct degrees $d_{u}$ for $u \in V(\operatorname{SSL}(n))$ is $\{3,4,6\}$. The following table give the edge partition of $G$.

The ABC and GA indices of star of silicate network are computed in the next two theorems.

Theorem 9. For $n \geq 3$,

$$
\begin{aligned}
\operatorname{ABC}(G)= & \left(12 \sqrt{\frac{5}{2}}+12 \sqrt{\frac{7}{2}}\right) n^{2}+\left(12 \sqrt{\frac{5}{3}}+24 \sqrt{\frac{1}{3}}+12 \sqrt{\frac{3}{2}}-20 \sqrt{\frac{7}{2}}-24 \sqrt{\frac{5}{2}}\right) n \\
& +\left(4-6 \sqrt{\frac{5}{3}}-24 \sqrt{\frac{1}{3}}-15 \sqrt{\frac{3}{2}}+12 \sqrt{\frac{7}{2}}+16 \sqrt{\frac{5}{2}}\right) \approx 41.4236 n^{2}-31.3186 n+11.7746
\end{aligned}
$$

Proof. We use the information given in Table 5 to compute the $\mathrm{ABC}$ index of star of silicate network as follows:

$$
\operatorname{ABC}(G)=\sum_{u v \in E(G)} \sqrt{\frac{d_{u}+d_{v}-2}{d_{u} d_{v}}} .
$$



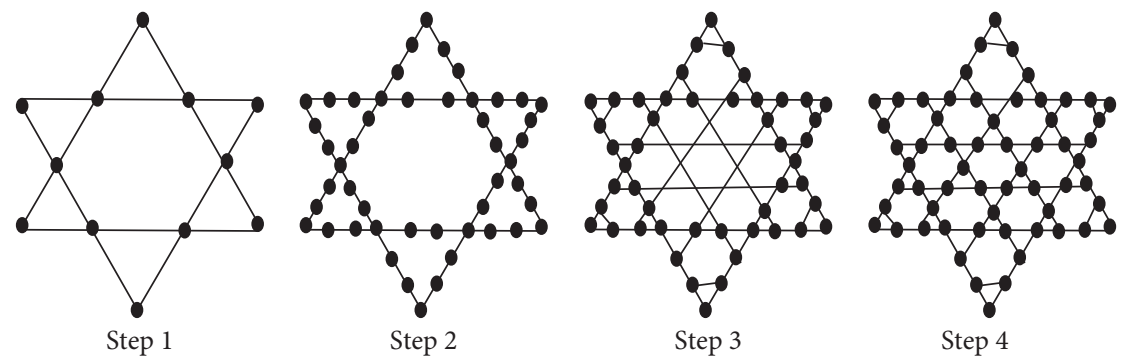

Figure 3: The construction of star of David graph of dimension 2.

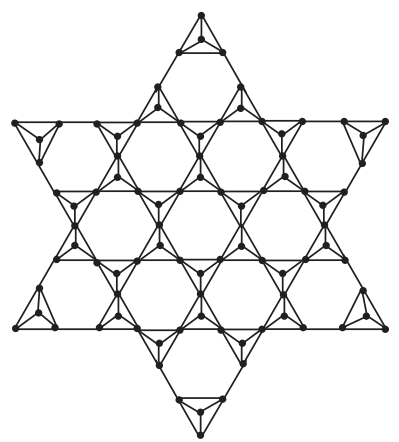

FIgURE 4: The graph of star of silicate network SSL(2).

TABLE 5: Edge partition of SSL $(n)$ centred on the degree of the end vertices of each edge, where $u v \in E(G)$.

\begin{tabular}{lc}
\hline$\left(d_{u}, d_{v}\right)$ & Number of edges \\
\hline$(3,3)$ & 6 \\
$(3,4)$ & $12(2 n-1)$ \\
$(3,6)$ & $\left(36 n^{2}-60 n+36\right)$ \\
$(4,4)$ & $(24 n-30)$ \\
$(4,6)$ & $24(n-1)$ \\
$(6,6)$ & $\left(36 n^{2}-72 n+48\right)$ \\
\hline
\end{tabular}

This implies that

$$
\begin{aligned}
\mathrm{ABC}(G)= & 6 \sqrt{\frac{3+3-2}{3 \cdot 3}}+12(2 n-1) \sqrt{\frac{3+4-2}{3 \cdot 4}}+\left(36 n^{2}-60 n+36\right) \sqrt{\frac{3+6-2}{3 \cdot 6}}+(24 n-30) \sqrt{\frac{4+4-2}{4 \cdot 4}} \\
& +24(n-1) \sqrt{\frac{4+6-2}{4 \cdot 6}}+\left(36 n^{2}-72 n+48\right) \sqrt{\frac{6+6-2}{6 \cdot 6}}
\end{aligned}
$$

By further simplification, we get the following form:

$$
\begin{aligned}
\mathrm{ABC}(G)= & \left(12 \sqrt{\frac{5}{2}}+12 \sqrt{\frac{7}{2}}\right) n^{2}+\left(12 \sqrt{\frac{5}{3}}+24 \sqrt{\frac{1}{3}}+12 \sqrt{\frac{3}{2}}-20 \sqrt{\frac{7}{2}}-24 \sqrt{\frac{5}{2}}\right) n \\
& +\left(4-6 \sqrt{\frac{5}{3}}-24 \sqrt{\frac{1}{3}}-15 \sqrt{\frac{3}{2}}+12 \sqrt{\frac{7}{2}}+16 \sqrt{\frac{5}{2}}\right) \approx 41.4236 n^{2}-31.3186 n+11.7746 .
\end{aligned}
$$


Theorem 10. For $n \geq 3, G A(G)=(72 \sqrt{2}+72 \sqrt{3}) n^{2}+((96$ $\sqrt{3} / 7)+(96 \sqrt{3} / 5)-72 \sqrt{2}-144 \sqrt{3}) n+((12 \sqrt{3} / 2)-(48 \sqrt{3}$ /7) $-(96 \sqrt{3} / 5)+12 \sqrt{2}+96 \sqrt{3}) \approx 226.5310 n^{2}-294.2294 n+$ 148.5074 .

Proof. The information in Table 5 and the expression $\sum_{u v \in E(G)} 2\left(\sqrt{d_{u} d_{v}} /\left(d_{u}+d_{v}\right)\right)$ yields the required result.

The central vertices of the tetrahedron lying on the corner has degree sum 11 . The central vertices of the tetrahedron that are adjacent to the vertices of degree 4 has degree sum 16. The central vertices of the remaining tetrahedron has degree sum 18. The vertices of degree 4 of the tetrahedron lying at corners has degree sum 14 . The 4 degree vertices that are adjacent to 6 degree vertices has degree sum
19. The remaining 4 degree vertices has degree sum 17 . The vertices of degree 6 have three kinds of degree sum. The vertices adjacent to one vertex of degree 4 have degree sum 26 , the vertices adjacent to two vertices of degree 4 have degree sum 28, and the remaining vertices have degree sum 30. Thus, the set of all distinct degree sums $S_{u}$ for $u \in V(\operatorname{SSL}(n))$ is $\{11,14,16,17,18,19,26,28,30\}$. From the above information and the construction of the graph, the edge partition is calculated as follows.

The $\mathrm{ABC}_{4}$ and $\mathrm{GA}_{5}$ of star of silicate network SSL $(n)$ are given in the following two results.

Theorem 11. The index $A B C_{4}$ of $G$, for $n \geq 3$, is computed as

$$
\begin{aligned}
\mathrm{ABC}_{4}(G)= & \left(12 \sqrt{\frac{7}{78}}+8 \sqrt{\frac{23}{30}}+36 \sqrt{\frac{1}{130}}+\frac{30}{13 \sqrt{2}}+\frac{6 \sqrt{29}}{5 \sqrt{2}}\right) n^{2} \\
& +\left(24 \sqrt{\frac{29}{238}}+\frac{96 \sqrt{2}}{17}+24 \sqrt{\frac{41}{442}}+12 \sqrt{\frac{19}{182}}-48 \sqrt{\frac{7}{78}}-16 \sqrt{\frac{23}{30}}-144 \sqrt{\frac{1}{130}}-\frac{120}{13 \sqrt{2}}+\frac{18 \sqrt{3}}{7 \sqrt{2}}+24 \sqrt{\frac{1}{15}}+8 \sqrt{\frac{11}{14}}-\frac{12 \sqrt{29}}{5 \sqrt{2}}\right) n \\
& +\left(\begin{array}{c}
\frac{12 \sqrt{5}}{11}+24 \sqrt{\frac{23}{154}}+\frac{6 \sqrt{13}}{7 \sqrt{2}}+12 \sqrt{\frac{1}{14}}+3 \sqrt{\frac{33}{19}}+12 \sqrt{\frac{43}{494}}+6 \sqrt{\frac{5}{13}}+18 \sqrt{\frac{5}{133}}+3 \sqrt{\frac{3}{2}}-36 \sqrt{\frac{29}{238}}-\frac{240 \sqrt{2}}{17} \\
-48 \sqrt{\frac{41}{442}}-24 \sqrt{\frac{19}{182}}+48 \sqrt{\frac{7}{78}}+8 \sqrt{\frac{23}{30}}+144 \sqrt{\frac{1}{130}}+\frac{120}{13 \sqrt{2}}-\frac{27 \sqrt{3}}{7 \sqrt{2}}-36 \sqrt{\frac{1}{15}}-12 \sqrt{\frac{11}{14}}+\frac{6 \sqrt{29}}{5 \sqrt{2}}
\end{array}\right) \\
\approx & 23.3735 n^{2}-58.4777 n+14.6962 .
\end{aligned}
$$

Proof. We use the information given in Table 6 to compute the formula for $\mathrm{ABC}_{4}$ index for $G$ as follows:

$$
\mathrm{ABC}_{4}(G)=\sum_{u v \in E(G)} \sqrt{\frac{S_{u}+S_{v}-2}{S_{u} S_{v}}} .
$$

This implies that

$$
\begin{aligned}
& \mathrm{ABC}_{4}(\operatorname{SSL}(n))=6 \sqrt{\frac{11+11-2}{11 \cdot 11}}+24 \sqrt{\frac{11+14-2}{11 \cdot 14}}+6 \sqrt{\frac{14+14-2}{14 \cdot 14}}+(24 n-36) \sqrt{\frac{14+17-2}{14 \cdot 17}} \\
& +(12 n-24) \sqrt{\frac{14+26-2}{14 \cdot 26}}+12 \sqrt{\frac{16+19-2}{16 \cdot 19}}+12 \sqrt{\frac{16+26-2}{16 \cdot 26}}+12 \sqrt{\frac{16+28-2}{16 \cdot 28}}+(24 n-60) \sqrt{\frac{17+17-2}{17 \cdot 17}} \\
& +12 \sqrt{\frac{17+19-2}{17 \cdot 19}}+(24 n-48) \sqrt{\frac{17+26-2}{17 \cdot 26}}+\left(12 n^{2}-48 n+48\right) \sqrt{\frac{18+26-2}{18 \cdot 26}}+(24 n-36) \sqrt{\frac{18+28-2}{18 \cdot 28}} \\
& +\left(24 n^{2}-48 n+24\right) \sqrt{\frac{18+30-2}{18 \cdot 30}}+12 \sqrt{\frac{19+26-2}{19 \cdot 26}}+12 \sqrt{\frac{19+28-2}{19 \cdot 28}}+\left(6 n^{2}-24 n+24\right) \sqrt{\frac{26+26-2}{26 \cdot 26}} \\
& +12 \sqrt{\frac{26+28-2}{26 \cdot 28}}+\left(12 n^{2}-48 n+48\right) \sqrt{\frac{26+30-2}{26 \cdot 30}}+(12 n-18) \sqrt{\frac{28+28-2}{28 \cdot 28}}+(24 n-36) \sqrt{\frac{28+30-2}{28 \cdot 30}} \\
& +\left(18 n^{2}-36 n+18\right) \sqrt{\frac{30+30-2}{30 \cdot 30}} \text {. }
\end{aligned}
$$


TABLE 6: Edge partition of SSL $(n)$ centred on the number of the vertices of the degree lying at the unit distance from the end vertices of each edge.

\begin{tabular}{lc}
\hline$\left(S_{u}, S_{v}\right)$ where $u v \in E(G)$ & Number of edges \\
\hline$(11,11)$ & 6 \\
$(11,14)$ & 6 \\
$(14,14)$ & $(24 n-36)$ \\
$(14,17)$ & $(12 n-24)$ \\
$(14,26)$ & 12 \\
$(16,19)$ & 12 \\
$(16,26)$ & 12 \\
$(16,28)$ & $(24 n-60)$ \\
$(17,17)$ & 12 \\
$(17,19)$ & $(24 n-48)$ \\
$(17,26)$ & $\left(12 n^{2}-48 n+48\right)$ \\
$(18,26)$ & $(24 n-36)$ \\
$(18,28)$ & $\left(24 n^{2}-48 n+24\right)$ \\
$(18,30)$ & 12 \\
$(19,26)$ & 12 \\
$(19,28)$ & $\left(6 n^{2}-24 n+24\right)$ \\
$(26,26)$ & 12 \\
$(26,28)$ & $\left(12 n^{2}-48 n+48\right)$ \\
$(26,30)$ & $(12 n-18)$ \\
$(28,28)$ & $(24 n-36)$ \\
$(28,30)$ & $\left(18 n^{2}-36 n+18\right)$ \\
$(30,30)$ & \\
\hline
\end{tabular}

After a simple calculation, the above equation can be reduced as follows:

$$
\left.\begin{array}{rl}
\mathrm{ABC}_{4}(G)= & \left(12 \sqrt{\frac{7}{78}}+8 \sqrt{\frac{23}{30}}+36 \sqrt{\frac{1}{130}}+\frac{30}{13 \sqrt{2}}+\frac{6 \sqrt{29}}{5 \sqrt{2}}\right) n^{2} \\
& +\left(24 \sqrt{\frac{29}{238}}+\frac{96 \sqrt{2}}{17}+24 \sqrt{\frac{41}{442}}+12 \sqrt{\frac{19}{182}}-48 \sqrt{\frac{7}{78}}-16 \sqrt{\frac{23}{30}}-144 \sqrt{\frac{1}{130}}-\frac{120}{13 \sqrt{2}}+\frac{18 \sqrt{3}}{7 \sqrt{2}}+24 \sqrt{\frac{1}{15}}+8 \sqrt{\frac{11}{14}}-\frac{12 \sqrt{29}}{5 \sqrt{2}}\right) n \\
& +\left(\begin{array}{l}
\frac{12 \sqrt{5}}{11}+24 \sqrt{\frac{23}{154}}+\frac{6 \sqrt{13}}{7 \sqrt{2}}+12 \sqrt{\frac{1}{14}}+3 \sqrt{\frac{33}{19}}+12 \sqrt{\frac{43}{494}}+6 \sqrt{\frac{5}{13}}+18 \sqrt{\frac{5}{133}}+3 \sqrt{\frac{3}{2}}-36 \sqrt{\frac{29}{238}} \\
-\frac{17}{17}-48 \sqrt{\frac{41}{442}}-24 \sqrt{\frac{19}{182}}+48 \sqrt{\frac{7}{78}}+8 \sqrt{\frac{23}{30}}+144 \sqrt{\frac{1}{130}}+\frac{120}{13 \sqrt{2}}-\frac{27 \sqrt{3}}{7 \sqrt{2}}-36 \sqrt{\frac{1}{15}}-12 \sqrt{\frac{11}{14}}+\frac{6 \sqrt{29}}{5 \sqrt{2}}
\end{array}\right) \\
\approx & 23.3735 n^{2}-58.4777 n+14.6962 .
\end{array}\right)
$$


Theorem 12. For $n \geq 3$, we have

$$
\left.\begin{array}{rl}
\mathrm{GA}_{5}(G)= & \left(24+\frac{36 \sqrt{13}}{11}+6 \sqrt{15}+\frac{6 \sqrt{195}}{7}\right) n^{2} \\
& +\left(\frac{48 \sqrt{238}}{31}+\frac{48 \sqrt{442}}{43}+\frac{6 \sqrt{91}}{5}-\frac{144 \sqrt{13}}{11}-12 \sqrt{15}-\frac{24 \sqrt{195}}{7}+\frac{48 \sqrt{210}}{29}+\frac{144 \sqrt{14}}{23}-24\right) n \\
& +\left(\begin{array}{l}
\frac{48 \sqrt{154}}{25}+\frac{2 \sqrt{323}}{3}+\frac{8 \sqrt{182}}{9}+\frac{96 \sqrt{19}}{35}+\frac{8 \sqrt{494}}{15}+\frac{16 \sqrt{26}}{7} \\
+\frac{48 \sqrt{133}}{47}+\frac{48 \sqrt{7}}{11}-\frac{72 \sqrt{238}}{31}-\frac{96 \sqrt{442}}{43}-\frac{12 \sqrt{91}}{5}+\frac{144 \sqrt{13}}{11}+6 \sqrt{15}+\frac{24 \sqrt{195}}{7}-\frac{72 \sqrt{210}}{29}-\frac{216 \sqrt{14}}{23}-30
\end{array}\right) \\
\approx & 71.0072 n^{2}-106.1903 n+20.7293 .
\end{array}\right)
$$

Proof. Using the information given in Table 6 and the formula for $\mathrm{GA}_{5}$, we get the proof.

\section{Rhenium Trioxide Lattice}

We review the degree-based topological descriptors for the $\mathrm{RO}(p, q, r)$ rhenium trioxide lattice in this section. [28]. It consists of rhenium atoms and oxygen atoms and is an inorganic compound. Rhenium trioxide is a red solid with a metallic cluster and a primitive cubic unit cell forming a crystal, that is, the $\mathrm{ReO} 3$ unit cell. Rhenium atoms are the vertices marked in a hollow circle, and oxygen atoms are the vertices identified in the solid circles. The unit cell $\mathrm{ReO} 3$ includes oxygen atoms of 12 and rhenium atoms of 8 (Figure 5).

By $\mathrm{ReO}_{3}$, we represent $p \times q \times r \mathrm{ReO}_{3}$ lattice. It is a threedimensional sequence of $p$ unit cell rows along the $X$-axis, $q$ unit cell columns along the $Y$-axis, and $r$ unit cell pages along the $Z$-axis.

The $\mathrm{RO}(p, q, r)$ graph is the $\mathrm{RO}(p, q, r)$ rhenium trioxide graph, which is constructed as follows:
Step 1: we draw a $3 d$-grid $M(p, q, r)$, which is described by the Cartesian product of paths $P_{p} \square P_{q} \square P_{r}$. Such vertices refer to atoms of rhenium.

Step 2: subdivide each of the $M(p, q, r)$ edges in this step. Oxygen atoms correspond to the new vertices. We are now going to mark the vertices of $\operatorname{RO}(p, q, r)$. The rhenium atoms will obtain the same $3 d$-grid mark as the vertices. Between two rhenium atoms $\left(a^{\prime}, b^{\prime}, c^{\prime}\right)$ and $\left(a^{\prime \prime}, b^{\prime \prime}, c^{\prime \prime}\right)$, the oxygen atom will receive the label $(a, b, c)$, where $a=\left(a^{\prime}+a^{\prime \prime} / 2\right), b=\left(b^{\prime}+b^{\prime \prime} / 2\right)$, and $c=\left(c^{\prime}+c^{\prime \prime} / 2\right)$. Two vertices $\left(x^{\prime}, y^{\prime}, z^{\prime}\right)$ and $\left(x^{\prime \prime}, y^{\prime \prime}\right.$, $\left.z^{\prime \prime}\right)$ are adjacent if $\mid\left(x^{\prime}-x^{\prime \prime}\right)+\left(y^{\prime}-y^{\prime \prime}\right)+\left(z^{\prime}-\right.$ $\left.z^{\prime \prime}\right) \mid=(1 / 2)$. The number of vertices and edges in $R O(p, q, r)$ are $4 p q r-p q-q r-p r$ and $6 p q r-$ $2 p q-2 q r-2 p r$, respectively. Throughout this section, $G$ denotes the graph of rhenium trioxide $\operatorname{RO}(p, q, r)$.

It follows from the construction of $\operatorname{RO}(p, q, r))$ that the degree of each vertex can belong to the set $\{2,3,4,5,6\}$.

We will obtain the $\mathrm{ABC}$ and $\mathrm{GA}$ indices of rhenium trioxide lattice in the following two results.

Theorem 13. For $p, q, r \geq 4$, the $A B C$ index of the graph $G$ is

$$
\begin{aligned}
\operatorname{ABC}(G)= & 6 \sqrt{\frac{1}{2}} p q r-2 \sqrt{\frac{1}{2}} p q-2 \sqrt{\frac{1}{2}} q r-2 \sqrt{\frac{1}{2}} p r+\left(16 \sqrt{\frac{1}{2}}-40 \sqrt{\frac{1}{2}}+24 \sqrt{\frac{1}{2}}\right) p+\left(16 \sqrt{\frac{1}{2}}-40 \sqrt{\frac{1}{2}}+24 \sqrt{\frac{1}{2}}\right) q \\
& +\left(16 \sqrt{\frac{1}{2}}-40 \sqrt{\frac{1}{2}}+24 \sqrt{\frac{1}{2}}\right) r+\left(24 \sqrt{\frac{1}{2}}-96 \sqrt{\frac{1}{2}}+120 \sqrt{\frac{1}{2}}-48 \sqrt{\frac{1}{2}}\right) \approx 4.2426 p q r \\
& -1.4142(p q+q r+p r) .
\end{aligned}
$$




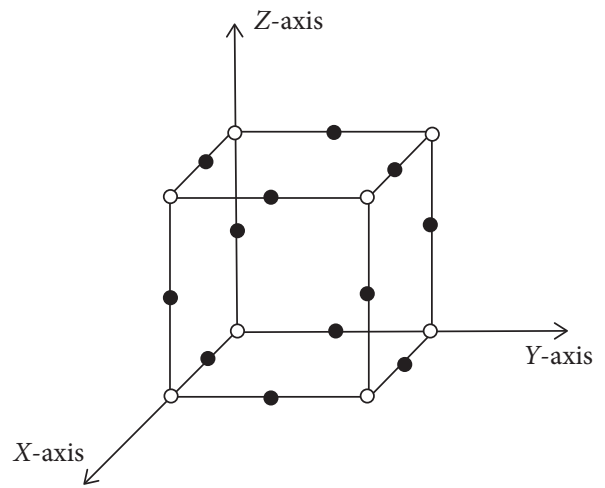

FIgure 5: The unit cell of rhenium trioxide lattice $\mathrm{ReO}_{3}$.

Proof. Using the information given in Table 7, the $\mathrm{ABC}$ index of rhenium trioxide lattice is calculated as follows:

$$
\operatorname{ABC}(G)=\sum_{u v \in E(G)} \sqrt{\frac{d_{u}+d_{v}-2}{d_{u} d_{v}}} .
$$

This implies that
TABLE 7: Edge partition of $\mathrm{RO}(p, q, r)$ centered on degree of final (end) vertices of each edge, where $u v \in E(G)$.

\begin{tabular}{lc}
\hline$\left(d_{u}, d_{v}\right)$ & Number of edges \\
\hline$(2,3)$ & 24 \\
$(2,4)$ & $16(p+q+r-6)$ \\
$(2,5)$ & $10(p q+q r+p r-4 p-4 q-4 r+12)$ \\
$(2,6)$ & $6(p q r-2 p q-2 q r-2 p r+4 p+4 q+4 r-8)$ \\
\hline
\end{tabular}

$$
\begin{aligned}
\mathrm{ABC}(G)= & 24 \sqrt{\frac{2+3-2}{2 \cdot 3}}+16(p+q+r-6) \sqrt{\frac{2+4-2}{2 \cdot 4}}+10(p q+q r+p r-4 p-4 q-4 r+12) \sqrt{\frac{2+5-2}{2 \cdot 5}} \\
& +6(p q r-2 p q-2 q r-2 p r+4 p+4 q+4 r-8) \sqrt{\frac{2+6-2}{2 \cdot 6}}
\end{aligned}
$$

After simplification, we obtain

$$
\begin{aligned}
\operatorname{ABC}(G)= & 6 \sqrt{\frac{1}{2}} p q r-2 \sqrt{\frac{1}{2}} p q-2 \sqrt{\frac{1}{2}} q r-2 \sqrt{\frac{1}{2}} p r+\left(16 \sqrt{\frac{1}{2}}-40 \sqrt{\frac{1}{2}}+24 \sqrt{\frac{1}{2}}\right) p+\left(16 \sqrt{\frac{1}{2}}-40 \sqrt{\frac{1}{2}}+24 \sqrt{\frac{1}{2}}\right) q \\
& +\left(16 \sqrt{\frac{1}{2}}-40 \sqrt{\frac{1}{2}}+24 \sqrt{\frac{1}{2}}\right) r+\left(24 \sqrt{\frac{1}{2}}-96 \sqrt{\frac{1}{2}}+120 \sqrt{\frac{1}{2}}-48 \sqrt{\frac{1}{2}}\right) \\
\approx & 4.2426 p q r-1.4142(p q+q r+p r) .
\end{aligned}
$$

Theorem 14. For $p, q, r \geq 4$,

$$
\begin{aligned}
\mathrm{GA}(G)= & \left(\frac{3 \sqrt{3}}{2}\right) p q r+\left(\frac{10 \sqrt{10}}{7}-3 \sqrt{3}\right) p q+\left(\frac{10 \sqrt{10}}{7}-3 \sqrt{3}\right) q r+\left(\frac{10 \sqrt{10}}{7}-3 \sqrt{3}\right) p r \\
& +\left(\frac{16 \sqrt{2}}{3}-\frac{40 \sqrt{10}}{7}+6 \sqrt{3}\right) p+\left(\frac{16 \sqrt{2}}{3}-\frac{40 \sqrt{10}}{7}+6 \sqrt{3}\right) q+\left(\frac{16 \sqrt{2}}{3}-\frac{40 \sqrt{10}}{7}+6 \sqrt{3}\right) r \\
& +\left(\frac{48 \sqrt{6}}{5}-32 \sqrt{2}+\frac{120 \sqrt{10}}{7}-12 \sqrt{3}\right) \approx 2.5980 p q r-0.6786(p q+q r+p r)-0.1353(p+q+r)+11.6862 .
\end{aligned}
$$


Proof. The proof follows by the information given in Table 7 and $\sum_{u v \in E(G)} 2\left(\sqrt{d_{u} d_{v}} /\left(d_{u}+d_{v}\right)\right)$.

From the construction of $\operatorname{RO}(p, q, r))$, it follows that the degree sum of each vertex can belong to the set $\{6,7,8,9,10,11,12\}$. Thus, the edge partition of $G$ is given below.
The $\mathrm{ABC}_{4}$ and $\mathrm{GA}_{5}$ indices of the rhenium trioxide lattice have been computed in the following two theorems.

Theorem 15. For $p, q, r \geq 4$, the $A B C_{4}$ of $G$ is computed as

$$
\begin{aligned}
\mathrm{ABC}_{4}(G)= & \sqrt{\frac{11}{2}} p q r+\left(\frac{12 \sqrt{2}}{5}+2 \sqrt{\frac{19}{110}}-\frac{7 \sqrt{11}}{3 \sqrt{2}}\right) p q+\left(\frac{12 \sqrt{2}}{5}+2 \sqrt{\frac{19}{110}}-\frac{7 \sqrt{11}}{3 \sqrt{2}}\right) q r+\left(\frac{12 \sqrt{2}}{5}+2 \sqrt{\frac{19}{110}}-\frac{7 \sqrt{11}}{3 \sqrt{2}}\right) p r \\
& +\left(2 \sqrt{\frac{7}{2}}+\frac{4 \sqrt{15}}{3 \sqrt{2}}+\frac{8 \sqrt{17}}{3 \sqrt{10}}-12 \sqrt{2}-8 \sqrt{\frac{19}{110}}+\frac{16 \sqrt{11}}{3 \sqrt{2}}\right) p+\left(2 \sqrt{\frac{7}{2}}+\frac{4 \sqrt{15}}{3 \sqrt{2}}+\frac{8 \sqrt{17}}{3 \sqrt{10}}-12 \sqrt{2}-8 \sqrt{\frac{19}{110}}+\frac{16 \sqrt{11}}{3 \sqrt{2}}\right) q \\
& +\left(2 \sqrt{\frac{7}{2}}+\frac{4 \sqrt{15}}{3 \sqrt{2}}+\frac{8 \sqrt{17}}{3 \sqrt{10}}-12 \sqrt{2}-8 \sqrt{\frac{19}{110}}+\frac{16 \sqrt{11}}{3 \sqrt{2}}\right) r \\
& +\left(24 \sqrt{\frac{11}{42}}+48 \sqrt{\frac{13}{14}}-18 \sqrt{\frac{7}{2}}-8 \sqrt{\frac{15}{2}}-16 \sqrt{\frac{17}{10}}+\frac{216 \sqrt{2}}{5}+24 \sqrt{\frac{19}{110}}-12 \sqrt{\frac{11}{2}}\right) \\
\approx & 2.3452 p q r-1.2468(p q+q r+p r)+3.0824(p+q+r)+25.0171 .
\end{aligned}
$$

Proof. By using the information given in Table 8, the $\mathrm{ABC}_{4}$ index is calculated as follows.

The formula for $\mathrm{ABC}_{4}$ index for $G$ :

$$
\operatorname{ABC}_{4}(G)=\sum_{u v \in E(G)} \sqrt{\frac{S_{u}+S_{v}-2}{S_{u} S_{v}}} .
$$

This implies that

$$
\begin{aligned}
\mathrm{ABC}_{4}(G)= & 24 \sqrt{\frac{6+7-2}{6 \cdot 7}}+24 \sqrt{\frac{7+8-2}{7 \cdot 8}}+8(p+q+r-9) \sqrt{\frac{8+8-2}{8 \cdot 8}}+8(p+q+r-6) \sqrt{\frac{8+9-2}{8 \cdot 9}} \\
& +8(p+q+r-6) \sqrt{\frac{9+10-2}{9 \cdot 10}}+8(p q+q r+p r-5 p-5 q-5 r+18) \sqrt{\frac{10+10-2}{10 \cdot 10}} \\
& +2(p q+q r+p r-4 p-4 q-4 r+12) \sqrt{\frac{10+11-2}{10 \cdot 11}} \\
& +2(3 p q r-7 p q-7 q r-7 p r+16 p+16 q+16 r-36) \sqrt{\frac{12+12-2}{12 \cdot 12}} .
\end{aligned}
$$

After a simple calculation, the above equation can be reduced as

$$
\begin{aligned}
\mathrm{ABC}_{4}(G)= & \sqrt{\frac{11}{2}} p q r+\left(\frac{12 \sqrt{2}}{5}+2 \sqrt{\frac{19}{110}}-\frac{7 \sqrt{11}}{3 \sqrt{2}}\right) p q+\left(\frac{12 \sqrt{2}}{5}+2 \sqrt{\frac{19}{110}}-\frac{7 \sqrt{11}}{3 \sqrt{2}}\right) q r+\left(\frac{12 \sqrt{2}}{5}+2 \sqrt{\frac{19}{110}}-\frac{7 \sqrt{11}}{3 \sqrt{2}}\right) p r \\
& +\left(2 \sqrt{\frac{7}{2}}+\frac{4 \sqrt{15}}{3 \sqrt{2}}+\frac{8 \sqrt{17}}{3 \sqrt{10}}-12 \sqrt{2}-8 \sqrt{\frac{19}{110}}+\frac{16 \sqrt{11}}{3 \sqrt{2}}\right) p \\
& +\left(2 \sqrt{\frac{7}{2}}+\frac{4 \sqrt{15}}{3 \sqrt{2}}+\frac{8 \sqrt{17}}{3 \sqrt{10}}-12 \sqrt{2}-8 \sqrt{\frac{19}{110}}+\frac{16 \sqrt{11}}{3 \sqrt{2}}\right) q+\left(2 \sqrt{\frac{7}{2}}+\frac{4 \sqrt{15}}{3 \sqrt{2}}+\frac{8 \sqrt{17}}{3 \sqrt{10}}-12 \sqrt{2}-8 \sqrt{\frac{19}{110}}+\frac{16 \sqrt{11}}{3 \sqrt{2}}\right) r \\
& +\left(24 \sqrt{\frac{11}{42}}+48 \sqrt{\frac{13}{14}}-18 \sqrt{\frac{7}{2}}-8 \sqrt{\frac{15}{2}}-16 \sqrt{\frac{17}{10}}+\frac{216 \sqrt{2}}{5}+24 \sqrt{\frac{19}{110}}-12 \sqrt{\frac{11}{2}}\right) \\
\approx & 2.3452 p q r-1.2468(p q+q r+p r)+3.0824(p+q+r)+25.0171 .
\end{aligned}
$$


TABLE 8: Edge partition of $\mathrm{RO}(p, q, r)$ centered on vertices' degree sum which is present at distance unite from the final (end) vertices of each edge.

\begin{tabular}{lc}
\hline$\left(S_{u}, S_{v}\right)$ where $u v \in E(G)$ & Number of edges \\
\hline$(6,7)$ & 24 \\
$(7,8)$ & 24 \\
$(8,8)$ & $8(p+q+r-9)$ \\
$(8,9)$ & $8(p+q+r-6)$ \\
$(9,10)$ & $8(p+q+r-6)$ \\
$(10,10)$ & $8(p q+q r+p r-5 p-5 q-5 r+18)$ \\
$(10,11)$ & $2(p q+q r+p r-4 p-4 q-4 r+12)$ \\
$(12,12)$ & $2(3 p q r-7 p q-7 q r-7 p r+16 p+16 q+16 r-36)$ \\
\hline
\end{tabular}

Theorem 16. For $p, q, r \geq 4$, we have

$$
\begin{aligned}
\mathrm{GA}(G)= & 6 p q r+\left(\frac{4 \sqrt{110}}{21}-6\right) p q+\left(\frac{4 \sqrt{110}}{21}-6\right) q r+\left(\frac{4 \sqrt{110}}{21}-6\right) p r+\left(\frac{96 \sqrt{2}}{17}+\frac{48 \sqrt{10}}{19}-\frac{16 \sqrt{110}}{21}\right) p \\
& +\left(\frac{96 \sqrt{2}}{17}+\frac{48 \sqrt{10}}{19}-\frac{16 \sqrt{110}}{21}\right) q+\left(\frac{96 \sqrt{2}}{17}+\frac{48 \sqrt{10}}{19}-\frac{16 \sqrt{110}}{21}\right) r+\left(\frac{48 \sqrt{42}}{13}+\frac{32 \sqrt{14}}{5}-\frac{96 \sqrt{2}}{17}+\frac{48 \sqrt{10}}{19}+\frac{16 \sqrt{110}}{7}\right) \\
\approx & 6 p q r-4.0022(p q+q r+p r)+7.9841(p+q+r)+71.8510
\end{aligned}
$$

Proof. From Table 8 and formula $\mathrm{GA}_{5}(G)=\sum_{u v \in E(G)} 2\left(\sqrt{S_{u} S_{v}} / S_{u}+S_{v}\right)$, supply the proof of the statement.

\section{Conclusion}

A topological index is the numeric quantity of a graph that characterizes the structure of a graph. The topological indices and physico-chemical properties such as atom-bond connectivity (ABC), Randić, and geometric-arithmetic (GA) indices are of great importance in the QSAR/QSPR studies that are used to estimate chemical compound bioactivity. Graph theory has been found to be of great value in this field of study.

The degree-based molecular topological indices are analyzed in this paper for certain chemical networks. These networks include enhanced mesh, triangular mesh, star of silicate network, and rhenium trioxide lattice. For these groups of chemical networks, the analytical closed formulae are derived.

\subsection{Open Problems.}

(1) In future, it will be interesting to discuss the distance-based topological indices of these networks

(2) Due to wide application of topological indices in chemistry, it will be interesting to explore new chemical structures and study their mathematical properties

\section{Data Availability}

The data used to support the findings of this study are included within the article.

\section{Conflicts of Interest}

The authors declare that there are no conflicts of interest regarding the publication of this paper.

\section{Authors' Contributions}

All authors have contributed equally.

\section{Acknowledgments}

This work was supported by Educational Commission of Anhui Province of China in 2019. Research on image restoration of low rank constrained with sparse representation (no. KJ2019A0906). Q. Iqbal was supported by the National Academy of Higher Education Commission Pakistan, under the Start-Up Research Grant Program (Ref. no. 94/IPFPII(Batch-I)/SRGP/NAHE/HEC/2020/84).

\section{References}

[1] K. C. Das, A. Yurttas, M. Togan, A. Cevik, and I. Cangul, "The multiplicative Zagreb indices of graph operations," Journal of Inequalities and Applications, vol. 2013, no. 1, p. 90, 2013.

[2] H. Wiener, "Structural determination of paraffin boiling points," Journal of the American Chemical Society, vol. 69, no. 1, pp. 17-20, 1947.

[3] N. Jafari Rad, A. Jahanbani, and I. Gutman, "Zagreb energy and Zagreb Estrada index of graphs," MATCH Communications in Mathematical and in Computer Chemistry, vol. 79, pp. 371-386, 2018.

[4] K. Das and K. Xu, "On relation between Kirchhoff index, laplacian-energy-like invariant and laplacian energy of graphs," Bulletin of the Malaysian Mathematical Sciences Society, vol. 39, 2015. 
[5] I. Milovanović, E. Milovanović, E. Glogić, and M. Matejić, "On Kirchho? index, laplacian energy and their relations," MATCH Communications in Mathematical and in Computer Chemistry, vol. 81, pp. 405-418, 2019.

[6] B. Bozkurt, A. D. Maden, and B. Zhou, "Note on the distance energy of graphs," MATCH Communications in Mathematical and in Computer Chemistry, vol. 64, pp. 129-134, 2010.

[7] S. Alikhanil, R. Hasni, and N. E. Arif, "On the atom-bond connectivity index of some families of dendrimers," Journal of Computational and Theoretical Nanoscience, vol. 11, pp. 1-4, 2014.

[8] M. Bača, J. Horváthová, M. Mokrišová, and A. Suhányiovă, "On topological indices of fullerenes," Applied Mathematics and Computation, vol. 251, pp. 154-161, 2015.

[9] A. Q. Baig, M. Imran, and H. Ali, "Computing Omega, Sadhana and PI polynomials of benzoid carbon nanotubes, Opto electron," Advanced Materials - Rapid Communications, vol. 9, pp. 248-255, 2015.

[10] V. Lokesha, T. Deepika, P. S. Ranjini, and I. N. Cangul, "Operations of nano structures via SDD, ABC4 and GA5 indices," Applied Mathematics and Nonlinear Sciences, vol. 2, no. 1,2017

[11] A. Q. Baig, M. Imran, and H. Ali, "On topological indices of poly oxide, poly silicate, DOX and DSL networks," Canadian Journal of Chemistry, vol. 93, no. 7, 2015, in press.

[12] M. V. Diudea, I. Gutman, and J. Lorentz, Molecular Topology, Nova, Huntington, WV, USA, 2001.

[13] I. Gutman and O. E. Polansky, Mathematical Concepts in Organic Chemistry, Springer-Verlag, New York, NY, USA, 1986.

[14] E. Estrada, L. Torres, L. Rodríguez, and I. Gutman, "An atombond connectivity index: modelling the enthalpy of formation of alkanes," Indian Journal of Chemistry, vol. 37A, pp. 849855, 1998.

[15] D. V. B. Furtula, "Topological index based on the ratios of geometrical and arithmetical means of end-vertex degrees of edges," Journal of Mathematical Chemistry, vol. 46, pp. 1369-1376, 2009.

[16] M. Ghorbani and M. A. Hosseinzadeh, "Computing $\mathrm{ABC}_{4}$ index of nanostar dendrimers," Optoelectronics and Advanced Materials - Rapid Communications, vol. 4, pp. 1419-1422, 2010.

[17] A. Graovac, M. Ghorbani, and M. A. Hosseinzadeh, "Computing fifth geometric-arithmetic index for nanostar dendrimers," Journal of Mathematical Nanoscience, vol. 1, pp. 33-42, 2011.

[18] S. Hayat and M. Imran, "Computation of certain topological indices of nanotubes," Journal of Computational and Theoretical Nanoscience, vol. 12, no. 1, pp. 70-76, 2015.

[19] S. Hayat and M. Imran, "Computation of certain topological indices of nanotubes covered by $C_{5}$ and, $C_{7}$," Journal of Computational and Theoretical Nanoscience, vol. 12, pp. 553-541, 2015.

[20] S. Hayat and M. Imran, "On some degree based topological indices of certain nanotubes," Journal of Computational and Theoretical Nanoscience, vol. 12, no. 8, 2015, in press.

[21] S. Hayat and M. Imran, "Computation of topological indices of certain networks," Applied Mathematics and Computation, vol. 240, pp. 213-228, 2014.

[22] A. Iranmanesh and M. Zeraatkar, "Computing GA index for some nanotubes, optoelectron," Advanced Materials-Rapid Communications, vol. 4, pp. 1852-1855, 2010.

[23] W. Lin, J. Chen, Q. Chen, T. Gao, X. Lin, and B. Cai, "Fast computer search for trees with minimal $\mathrm{ABC}$ index based on tree degree sequences," MATCH Communications in Mathematical and in Computer Chemistry, vol. 72, pp. 699-708, 2014.

[24] Y. Shang, "Lower bounds for Gaussian Estrada index of graphs," Symmetry, vol. 10, no. 8, p. 325, 2018.

[25] Y. Shang, "On the number of spanning trees, the Laplacian eigenvalues, and the Laplacian Estrada index of subdividedline graphs," Open Mathematics, vol. 14, no. 1, pp. 641-648, 2016.

[26] K. Sathish and B. Rajan, "On strong metric dimension of certain triangular tessellations," International Journal of Pure and Applied Mathematics, vol. 101, no. 5, pp. 637-645, 2015.

[27] F. Simon Raj and A. George, "On the metric dimension of silicate stars," ARPN Journal of Engineering and Applied Sciences, vol. 10, no. 5, pp. 2187-2192, 2015.

[28] S. Stephena, B. Rajana, J. Ryan, C. Grigoriousc, and A. Williama, "Power domination in certain chemical structures," Journal of Discrete Algorithms (Amst), vol. 33, p. 1018, 2015. 